\title{
Methodical Scheme for Introduction of Combinatorial Compounds in Mathematics Education
}

\author{
Ivan Georgiev ${ }^{1}$, Ivo Andreev ${ }^{2}$ \\ ${ }^{I}$ Department of Applied Mathematics and Statistics, University of Ruse, 8 Studentska str. 7000 Ruse, Bulgaria \\ ${ }^{2}$ University of Ruse, 8 Studentska str. 7000 Ruse, Bulgaria
}

\begin{abstract}
The article is structured as follows. In section 1 , the Introduction, a historical summary on the arising of the combinatorics is given. Next, a short review of the part of the combinatorics in the teaching of mathematics in Bulgaria is presented. At the beginning of section 2, combinatorial compounds, included in the Mathematics curriculum in Bulgaria, are reviewed. A new methodical scheme for the introduction of basic combinatorial compounds is proposed. Then, an alternative approach for solving combinatorial problems in teaching and learning mathematics is analyzed in detail. The innovative methods are illustrated with four particular sample tasks in section 3. Certain conclusions on the specific issues are drawn at the end of the article.
\end{abstract}

Keywords - education, combinatorics, combinatorial compounds, teaching \& learning, problem solving.

\section{Introduction}

For centuries, humanity has been solving various combinatorial problems.

Towards the end of the sixteenth century, knowledge, concerning the properties of figured numbers, the construction of magic and other

DOI: 10.18421/TEM101-52

https://doi.org/10.18421/TEM101-52

Corresponding author: Ivo Andreev,

University of Ruse, 8 Studentska str. 7000 Ruse, Bulgaria.

Email: i.andreev2010@abv.bg

Received: 25 November 2020.

Revised: 01 February 2021.

Accepted: 12 February 2021.

Published: 27 February 2021.

(cc) BY-NC-ND (C) 2021 Ivan Georgiev \& Ivo Andreev; published by UIKTEN. This work is licensed under the Creative Commons Attribution-NonCommercial-NoDerivs 4.0 License.

The article is published with Open Access at www.temjournal.com numerical squares, as well as the characteristics of binominal coefficients, was gained. Thus, combinatorics came forward, as one of the oldest and most significant branches of mathematics. Initially, it was created as an option of calculating the chances of winning in different gambling games. Gradually, it attained serious significance for probability theory, control systems theory, statistics and other branches of science and technology.

The first scientific research in combinatorics belonged to the Italian scientists J. Tartano, N. Tartaglia (1499-1557), G. Galilei (1564-1642) and the French scientist B. Pascal (1623-1662) and P. Fermat [13].

The beginning of the combinatorics as a science was initiated in the eighteenth century. It is related to the emergence of probability theory and the necessity of calculating the number of different combinatorial configurations.

The term "combinatorics" was first introduced by the German mathematician Gottfried Wilhelm Leibniz (1646-1716), published in his scientific work "Dissertation on Combinatorial Art" in 1666.

L. Euler has made significant contribution to the development of combinatorics. The appearance and development of the computer technology has premised heightened interest and progress of combinatorics, due to the opportunity for solving complex and multidimensional [10], [11] combinatorial problems.

Nowadays, combinatorics is a branch of mathematics, in which the questions of choice and arrangement of the elements of finite sets, according to certain rules, are studied. It has a variety of methods for solving so many diverse problems, that it is difficult to define its limits.

If the purpose of studying combinatorics in a school course of Mathematics has to be defined briefly, it would be the development of the so-called "combinatorial thinking", which should also be developed in the future by Mathematics teachers. This problem has motivated the authors to write this article. 
In the modern teaching of Mathematics in Bulgaria, the number of hours devoted to the learning of combinatorics and probabilities, is too limited. Currently, the definitions of the combinatorial compounds and corresponding formulas for calculating their numbers are listed in the training. For learners, the direct introduction of these definitions causes numerous difficulties. The problem derives from the fact that they are often misled on which definition to use. Even though they are supported by a sufficient number of examples, students find it difficult to realize the connection between the combinatorial problem and the specific type of the compound. Thereby, they have troubles in defining the specific type of compounds and, respectively, their number.

In particular, the content of mathematics training includes a system of specific knowledge, skills and competencies, but there is no system of knowledge and their corresponding ways of logical thinking and explanation, and without them knowledge cannot be acquired and applied [2].

The article aims to present an alternative method for introducing fundamental concepts and calculating basal compounds of combinatorics in the school course in Mathematics. This method gives the opportunity, naturally, by asking and answering three consecutive questions, the specific types of compounds, as well as their numbers, to be properly determined. Thus, the introduction into definitions of the various combinatorial compounds takes place in parallel, through several stages, so that learners will be able to reach the type of the combinatorial compound in an easier and more intelligible way, going through the stages successively.

\section{Exposition and Methodology}

\subsection{Combinatorial Compounds in the Educational Content in our Country}

The question "When and how to conduct training in combinatorics and probability theory?" still rouses the interest of eminent experts in this particular subject, worldwide. At the primary stage of education, students are introduced to some types of combinatorial tasks; however, the material on combinatorics is studied more thoroughly, mainly in the secondary education [3], [4], [14]. Examining at length the content of each textbook in Mathematics, which contains material on combinatorics, it will be noticed that the place of this section of mathematics has been changing over the years.

In 1939, the curriculum of algebra in the secondary education in Bulgarian schools included the topics of combinatorics and probabilities [12].
After the Second World War, training in combinatorics collapsed. In 1945, the topics related to combinatorial compounds, binomial theorem and the primary knowledge of the probability theory were removed from the curriculum [12].

The reform in the mathematics education in the world had a real effect on the modernization of the curriculum in Bulgaria, only between the years 1963 and 1966 [12].

At present, one of the changes that have occurred in Mathematics curricula is the propaedeutic introduction of the concept of probability in the $6^{\text {th }}$ grade. A great number of combinatorial situations arise, however, they are relatively simple and estimating the number of favourable and all sorts of events is often largely intuitive.

From the 2017/2018 school year, according to the State educational requirements, combinatorial compounds are introduced in the 8th grade [6]. Before the start of the school year, the teacher is faced with the choice of a proper textbook and a publisher, and that has to be approved by the order of the Minister of Education and Science. Whatever textbook the teacher chooses, the introduction of concepts from combinatorics is put into practice in a similar way. For example, in [1], [5], [7], [8], [15], and [16] combinatorial compounds are defined as follows:

- Definition of variations without repetition. Variations without repetition of $\mathbf{n}$ elements of class $\boldsymbol{k}$ are called such compounds, each of which contains $\mathbf{k}$ different elements from the given $\mathbf{n}$ and differ from each other either in the elements or in the order of the elements.

- Definition of combinations without repetition. Combinations without repetition of $\mathbf{n}$ elements of class $\boldsymbol{k}$ are called such compounds, each of which contains $\mathbf{k}$ different elements of the given n and differ from each other by at least one element.

In previous mathematics curricula, definitions of compounds with repetitions are also given:

- Definition of variations with repetitions. Variations with repetitions of $\mathbf{n}$ types of elements of the k-th class are called ordered compounds, in which repetition of the elements up to the class of compounds is allowed [5], [15].

Therefore, two variations with repetition differ from each other (are different) if they have at least one element that participates a different number of times in the two, or if different elements stand in a position with the same number in both variations [5], [9], [15]. 
- Definition of combinations with repetitions. Combinations with repetitions of $\mathbf{n}$ types of different elements of $\mathbf{k}$-th class are called unordered compounds containing $\mathbf{k}$ elements, each of which is from the given $\mathbf{n}$ types of elements [5], [9], [15].

The definition clarifies that: the number of repetitions of the elements in the combinations does not exceed their class; two combinations of $\mathrm{k}$ elements of $n$-th class coincide if and only if both contain the same number of the same elements of the given $\mathrm{k}$ types, regardless of the order in which they are written [5].

In this way, the defined concepts cause confusion and misuse of different definitions. Learners often cannot recognize the correct compound by directly applying the definitions, especially after the introduction of iterative definitions, in which the number of elements in a class may exceed the number of elements in the set. The following task system will be considered as an example of such confusion.

Task 1. A football fan wants to win the jackpot by guessing the results of 13 football matches. How many forms does he have to fill in to make sure he guesses all the matches?

Here, learners often confuse the number of elements in a compound by assuming that it is 3 and the number of the set of elements, involved in the compound, is 13 . They do not take into account the fact that the compound is repetitive and its number of elements may exceed the number of elements of the set.

Given these vaguely structured definitions, an alternative method for the introduction of these four basic combinatorial compounds has been proposed. In this way, the difference between these compounds will become more clearly visible and, above all, understandable by the learners.

Most tasks are simple and the number of events is calculated directly. It is not necessary to introduce concepts from combinatorics in them. In the more complex ones, which are considered in the optional elective courses (OEC) or the compulsory elective courses (CEC) in mathematics, as well as in the preparation for the Mathematical Olympiad, for calculating certain probabilities, the number of events cannot be directly listed. The above statements can be illustrated by the following system task: A box with 10 balls of the same size and shape is given. The only difference is the colour. Of these 10 balls -8 are white and 2 black. What is the probability of randomly drawing two white balls without returning?
Students should calculate the number of possible and the number of favourable cases. Most often, this number is not so obvious and it is difficult for learners to count it directly. The teacher immediately sees the combinatorial problem and the need to introduce concepts from combinatorics, but such concepts are brought into use only in 10th grade. The teacher can help in the following way, without introducing concepts from combinatorics - for the total number of which two balls can be drawn; he can show the following sketch:

$$
\underbrace{\circ \circ \circ \circ \cdots}_{10} \circ
$$

and to ask how many ways the first ball can be taken out, suggesting that it can be taken out together with the second, the third, the fourth, ..., the tenth. Obviously the ways are 9 .

Then he asks how many ways the second ball can be taken out, suggesting that it can be taken out together with the third, fourth,..., tenth. The teacher emphasizes the fact that it has already been taken out with the first ball. The number is 8 .

Asking a third question about the third ball, again it is suggested that it can be taken out with the fourth, fifth, ..., tenth. There are already 7 ways. The teacher lays stress on the fact that so far the number is $9+8$ +7 , if you continue in the same way. Thinking similarly about the other balls, for the total number of ways, we get $9+8+7+\ldots+2+1=45$. For the favorable number out of 8 white balls to be drawn 2 white balls, students can now give the following answer: $7+6+\ldots+1=28$. Then the probability is

$$
\frac{7+6+\ldots+3+2+1}{9+8+\ldots+1}=\frac{28}{45} \text {. }
$$

In a similar way, the teacher would consider the more generalized problem with $\mathrm{n}+1$ white balls and 2 black ones, requiring the calculation of the following sum $1+2+3+\ldots+\mathrm{n}$. The last sum can be calculated by combining, according to the so-called Gaussian method, which some of the students get acquired with in an extracurricular Mathematics course.

\subsection{Exposition of an Alternative Approach to Solving Combinatorial Problems}

Figure 8 proposes another method for both introducing and calculating the basic elements of combinatorics.

The propaedeutics of combinatorial compounds in this approach are based mainly on asking questions and prompting their answers by the teacher. The concepts are built in parallel in several stages. The teacher can approach as follows, by drawing the following figure (Figure 1). 


$$
\underbrace{1,2,3, \ldots, \mathrm{n}}_{\text {number of elements }}
$$

Figure 1. A numbering of n balls

The teacher explains to the students that this is a box with balls of the same shape and weight. In order to distinguish one ball from another, a numbering of $1,2,3, \ldots, \mathrm{n}$ is given. The teacher says that if he takes a certain amount of balls out of the box, it is called a sample and asks the students the following question: How many different samples can be made? Some of them come to the conclusion that the question, put in this way, is incomplete. The teacher emphasizes the fact that the question posed like that is incomplete indeed and in order a correct assessment of the number of the samples, that can be taken, to be possible, clear criteria by which two samples will differ from each other have be defined. Then the teacher asks the students questions regarding these criteria by which the samples will differ from each other. They give different assumptions and some of them are correct. To settle the dispute, he gives the following example - if two people take out balls from two boxes of the same composition - the first takes out two balls and the second - three balls, are these two samples the same? Here the students are unanimous in the negative answer. Then the teacher emphasizes the fact that the first thing that can distinguish two samples is their volume.

\section{Sample \\ 1. Volume \\ 2. Arrangement \\ 3. Repeatability}

Figure 2. Three criteria for distinguish

The next question is asked by giving specific values of the elements in the box and the samples. There are 10 balls in the box, on which the numbers from 0 to 9 are written. If there are two such boxes, and one person draws balls with numbers 3 and 1 in succession, while another one draws 1 and 3 in succession from the other box, they both make a sample with a volume of 2 . Are these samples the same (Figure 3 and Figure 4)?

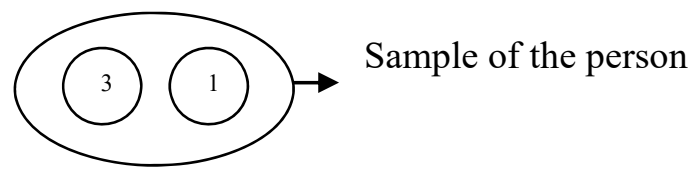

Figure 3. Sample of the person

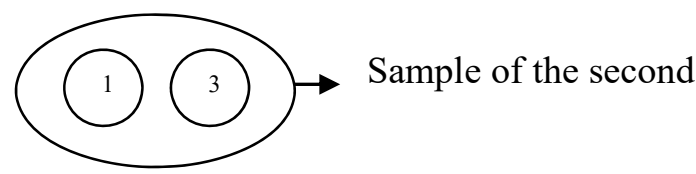

Figure 4. Sample of the second
Students have a different opinion on this issue. Some say the samples are different and others claim they are the same. The teacher intervenes again and explains that the question he asked is not complete. $\mathrm{He}$ did not say that in the specific sample the arrangement matters, i.e., if the places of two random balls in the sample are exchanged, would it remain the same or changes. The teacher explains that this depends on the nature of a specific combinatorial problem. His statement is illustrated by the following example: if these two people intended to make a twodigit number, then the two samples would be different - (the first would have made the number 31 and the second the number 13). If the numbers of the balls corresponded to a colour and it does not matter where that colour is in the sample, then the two samples would be the same.

The second main criterion, which is emphasized in the sample, is importance of the arrangement, i.e. if the order of two unspecified elements is changed, would the sample change too.

After clarifying these two criteria, the teacher asks the question: Is there another criterion by which the samples can be distinguished? Most students are of the opinion that there is not. Then, the provocative question in the numerical example with the numbering of the balls follows: Is it possible to draw two balls and with their numerical numbering to make the number 55, i.e. the sample should look like this (Figure 5):

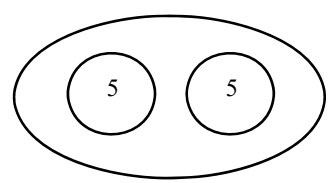

Figure 5. Possibility for repeatability of an element in the sample

Some students begin to realize that this is only possible if, after drawing the first ball, it returns to the box. The teacher confirms this and indicates the third criterion by which one sample can be distinguished from another, namely is it possible the same element to be found in several places in the sample. In the example with the box, would we return each drawn ball before drawing another or not.

Figure 2 gives the three main criteria by which we will distinguish one sample from another. This figure is completed sequentially after the establishment of each criterion. Returning to Figure 1, following the three criteria in Figure 2, the first of which is the sample volume and complements Figure 1 as follows:

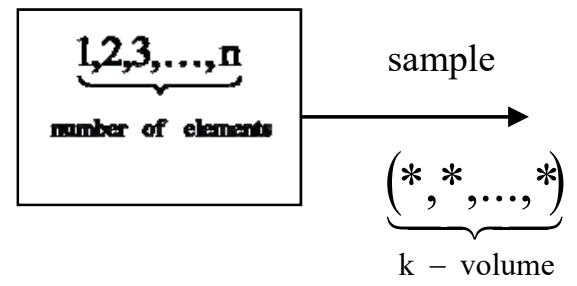

Figure 6. Sample with volume $\mathrm{k}$ 


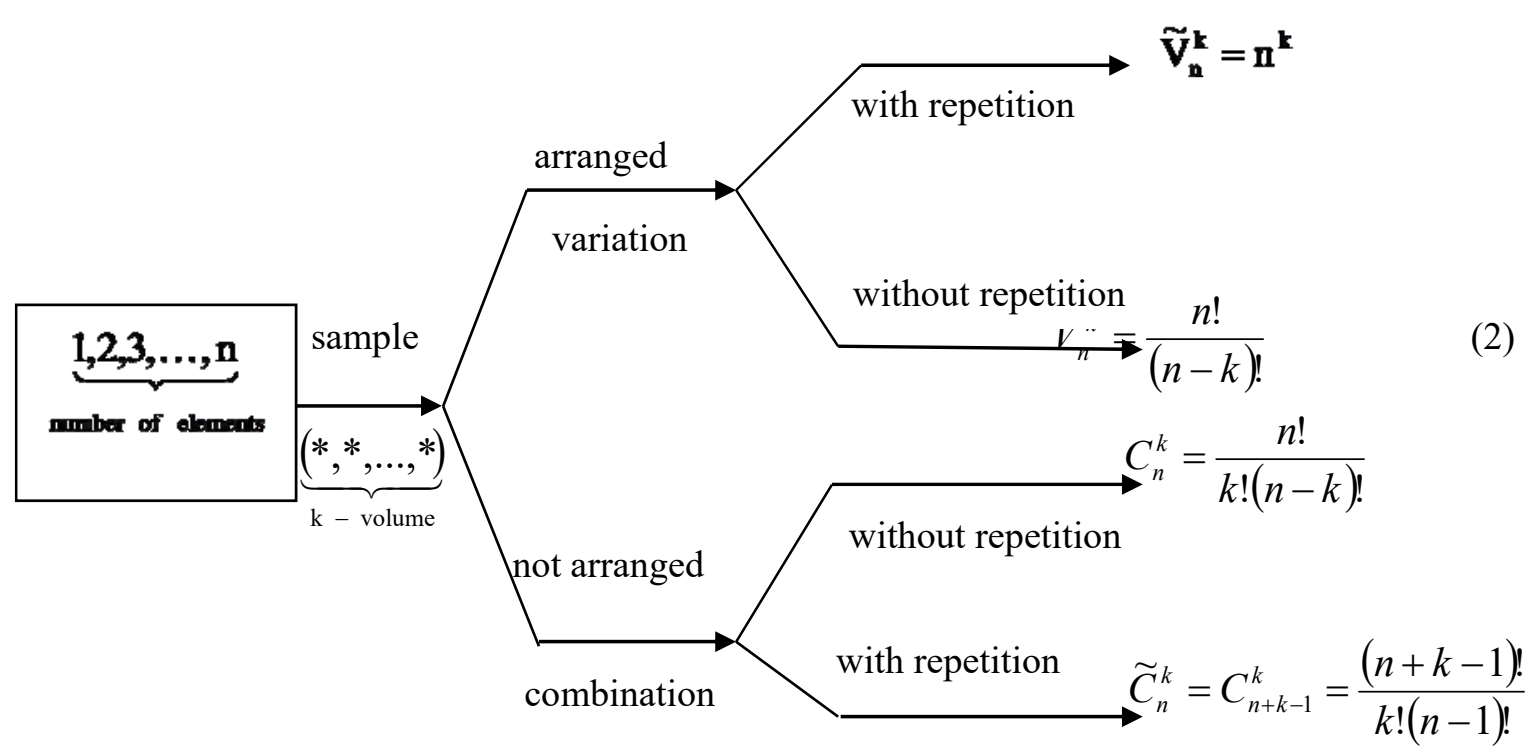

Figure 8. The sample can be with repetition or without repetition. Calculation of the basic elements of combinatorics

Then the teacher returns to Figure 2 and indicates the second criterion - an arrangement. Depending on whether the arrangement is relevant or not, Figure 6 will be divided into two main branches - in the first the arrangement is relevant and in the second - it is not (here Figure 6 is added to Figure 7).

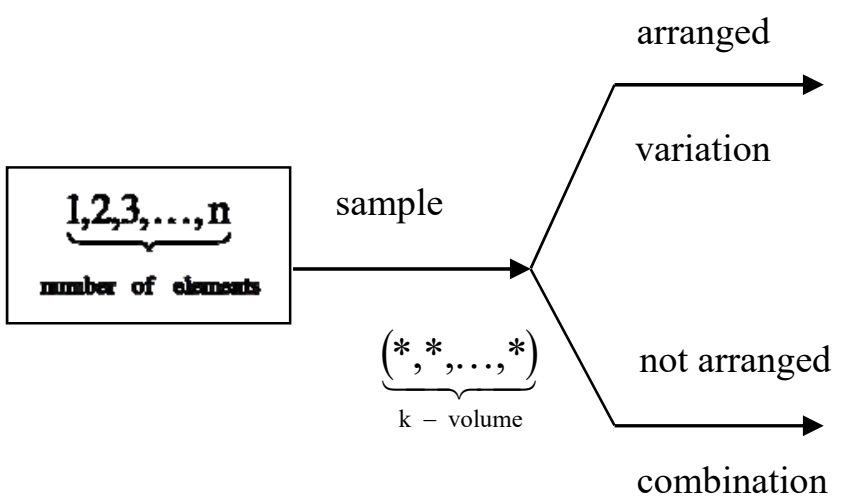

Figure 7. The sample can be arranged or not arrangea

Apart from the additional new branches, Figure 8 explains that when $\mathrm{n}$ elements are sampled with volume $\mathrm{k}$, in which case the arrangement matters, the term variation will be used, and if the arrangement does not matter, the term combination will be used. Figure 8 is completed with these two terms. The aim is for the students, following Figure 8, to summarize that the number of samples with volume $\mathrm{k}$, filled in by $\mathrm{n}$ elements, where the arrangement is relevant and we have allowed repetition, is calculated by the formula:

$$
\widetilde{\mathrm{V}}_{\mathrm{n}}^{\mathrm{k}}=\mathrm{n}^{\mathrm{k}}
$$

As writing formula (1) in front of the students, the teacher stresses on every symbol in it. Each symbol corresponds to Figure 8. The volume of the totality is
The third and last criterion - repeatability, is explained by Figure 2. According to this criterion, it is commented or analyzed to what extent the repetition of elements in the sample is allowed or not. Each of the two branches of Figure 7 divides into two more branches - in one of them the repetition of the elements is allowed, and in the other - it is not (here it is added Figure 7 to Figure 8).

$\mathrm{n}$; the volume of the sample is $\mathrm{k}$. The symbol $\mathrm{V}$ indicates that this is an arranged sample, and indicates that repetition is possible in the sample. The teacher does the same with the other three cases in Figure 8.

To answer the main question - What is the number of samples; it is necessary to describe precisely the criteria by which two samples will differ from one another:

1. Volume of the sample $\mathrm{k}$ and volume of the totality $\mathrm{n}$.

2. Arrangement in the sample (whether the change of the position of two elements changes the sample - yes / no).

3. Repeatability. We have to find out whether the same element can be in several places simultaneously in the sample - yes / no. 
Following the scheme, all three questions are answered consecutively. Thus, the question about the type and number of the sample is answered unambiguously, (variation with / without repetition, combination with / without repetition).

Only for compounds with repetitions (1) and (3) it is possible $\mathrm{k}>\mathrm{n}$.

In the special case, when $k=n$ is called permutation of $n$ elements and it is denoted by $\mathrm{P}_{\mathrm{n}}=\mathrm{n} !$.

Finally, the teacher should mention to the students that the four main types of combinatorial compounds not in the least exhaust the whole diversity of combinatorial configurations.

\section{Application of the Alternative Approach for Solving Combinatorial Compounds}

Task 2. How many different forms should we, at least, fill in the game TOTO 1 - 13 matches, to ensure that we will guess them all?

Answer: One specific prediction (form) consists of 13 matches, i.e. one sample has a volume $\mathrm{k}=13$.

The different elements that can stand in each of the 13 positions are three $(1, \mathrm{x}, 2)$, i.e. $n=3$.

Moving on Figure 8, we come to the arrangement matters. If we exchange the predictions of two different matches, the form changes, i.e. the arranged sample is permutation, by repeating the elements in the sample (for example, it is possible to mark all matches with $\mathrm{x}$ ). Moving consecutively along Figure 8 , it is found that $\mathrm{k}=13, \mathrm{n}=3$, variation, with repetition, i.e.:

$$
\widetilde{\mathrm{V}}_{3}^{13}=3^{13}=1594323 .
$$

The same problem can be solved by repeatedly applying the multiplication rule. $\underbrace{3.3 \ldots 3}_{13 \text { times }}$.

Task 3. For one race, a team of 12 people has to choose a captain, (the captain always plays,) and a reserve player. What is the number of different ways in which this can be done?

Answer: Looking at the diagram, first the volume of the sample is found. Two people have to be chosen, i.e. $k=2$, (each choice of two people is one sample). The volume of the totality is $n=12$ ( 1 of the 12 competitors can sit in each position in the sample).

№ -> serial number

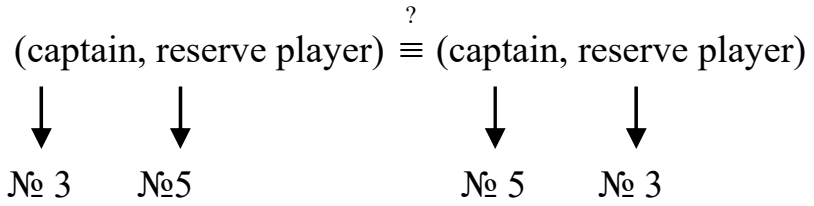

Figure 9. Recognition whether the sample is arranged or not arranged
In order to understand whether the sample is arranged, we play a specific variant, in which we exchange the places of two elements and ask the question whether a change has occurred (Figure 9). From Figure 9 it is clear that the arrangement matters, i.e. variation.

As for the question of repetition, a player № 5 cannot be both a captain and a reserve, i.e. variation without repetition:

$$
\mathrm{V}_{12}^{2}=\frac{12 !}{(12-2) !}=12.11=132 .
$$

Task 4. How many different forms from the TOTO 2 game (6 of 49) do we need to fill in to make sure we hit a six?

Answer: Again according to the scheme.

Each specific filled in form is one sample. Its volume is $\mathrm{k}=6$.

One of the $n=49$ numbers can stand in each of these positions. The arrangement does not matter, i.e. combination. It is not possible to draw the same number two or more times, i.e. combination without repetition:

$\mathrm{C}_{49}^{6}=\frac{49 !}{6 !(49-6) !}=\frac{49 \cdot 48 \cdot 47 \cdot 46 \cdot 45 \cdot 44}{6 \cdot 5 \cdot 4 \cdot 3 \cdot 2 \cdot 1}=13983816$.

Task 5. You want to buy five candies. The store has 3 different types: $x, y, z$. In how many ways can you do the purchase?

Answer: One specific purchase is one particular sample. For example, $(\mathrm{x}, \mathrm{x}, \mathrm{x}, \mathrm{y}, \mathrm{z})$, i.e. the sample consists of $k=5$ elements. At each position, out of the five, it is possible to stand $n=3$ candies of the type $\mathrm{x}, \mathrm{y}$ or $\mathrm{z}$. The arrangement in the sample does not matter, $(\mathrm{x}, \mathrm{x}, \mathrm{x}, \mathrm{y}, \mathrm{z})$ and $(\mathrm{x}, \mathrm{x}, \mathrm{x}, \mathrm{z}, \mathrm{y})$ are the same purchase, i.e. combination with repetition of an element in the sample. In the above examples, the element $\mathrm{x}$ is placed in three places:

$$
\widetilde{C}_{3}^{5}=C_{3+5-1}^{5}=C_{7}^{5}=C_{7}^{2}=\frac{7.6}{2.1}=21 .
$$

\section{Conclusion}

This article aims to build up a pedagogical approach to the introduction of basic combinatorial compounds.

The student has to answer three main questions:

- "How much?", determining the volume of the sample and the set from which it is made up;

- Arrangement, i.e. does the exchange of the position of two elements in the sample matter?

- Repeatability in the sample, i.e. can the same element stand at several positions at the same time?

Answering these questions consecutively, the students move along the branches of Figure 8. In this way, the learners, by themselves, easily find the specific type of compounds, as well as determine their number. 
With the so - proposed alternative approach (Figure 8) for the introduction of basic concepts of combinatorics, a greater clarity of the considered concepts is achieved.

The approach, considered in this way, allows the students, in spite of the limited number of hours, to build combinatorial habits, necessary for the correct solution of problems in combinatorics and probability theory, faster and more easily.

The presented material allows a conceptual apparatus to be formed, and the necessary knowledge of combinatorics to be given, in a slightly different and more visual way, by means of Figure 8 .

The approach stimulates the development of different mental processes in students, helps the upgrowth of skills and habits in self-reliant performance of various tasks.

Applying Figure 8, even with a small number of examples, it is enough to form combinatorial thinking in students. The article presents tasks from different areas of application of combinatorics, which are solved according to the propounded alternative approach in Figure 8.

The stated methodic and the provided methodical instructions for training the students in solving combinatorial problems can be applied not only in the primary, secondary schools, or in the extracurricular forms of work, but also in the universities, where combinatorial compounds are studied and used.

An experimental study concerning statistical comparison of the success of students in secondary education, by applying the alternative methodic for combinatorics training, is forthcoming.

\section{Acknowledgements}

This paper contains results of the work on project FNI5000, financed by "Scientific Research" Fund of Ruse University.

The authors acknowledge the significant contribution and helpful suggestions of Prof. Dr. Margarita Varbanova, which improved the quality of the paper.

This paper contains results of the work on project No 2021-FNSE-05, financed by "Scientific Research Fund" of Ruse University.

\section{References}

[1]. Badnjarević, D. (2015). Elementi verovatnoće u nastavi matematike u srednjoj školi i izrada elektronskog materijala. Retrieved from:

http://elibrary.matf.bg.ac.rs/bitstream/handle/1234567 89/3991/masDraganaBadnjarevic.pdf?sequence $=1$ [accessed: 10 October 2020].

[2]. Grozdev, S., Stoeva, T. \& Raeva, I. (2018). Problems and methodical solutions. Sofia: Association European Kangaroo. ISBN 978-6197020-25-0.

[3]. Karakoleva, S. \& Guteva, P. (2020). Use of Cloud Technologies for Training and test Control on the Topic "Basic Combinatorial Concepts" in Eighth Grade. Proceedings of University of Ruse-2020, vol.59, book 6.1, pp. 52-57.
[4]. Karakoleva, S. \& Ivanov, S. (2020). Use of Cloud Technologies for Training and Test Control on the Topic "Elements of Probabilities and Statistics" in Seventh Grade. Proceedings of University of Ruse2020, vol.59, book 6.1, pp. 58-63.

[5]. Kornilov, P., Nikulina, N. \& Semenova, O. (2005). Elements of discrete mathematics. Textbook. Yaroslavl: YAGPU named after K. D. Ushinsky. Retrieved from:

http://window.edu.ru/resource/283/65283/files/yspu01 .pdf [accessed: 15 October 2020].

[6]. Mathematics curriculum for $8^{\text {th }}$ grade. (2017). Mathematics curriculum for $8^{\text {th }}$ grade 2017/2018 school year. Retrieved from:

https://www.mon.bg/upload/13462/UP_8kl_Maths_Z P.pdf [accessed: 15 October 2020].

[7]. Paskaleva, Z., Alashka, M., Paskalev, P. \& Alashka, R. (2017). Mathematics for $8^{\text {th }}$ grade. Sofia: Arhimed, ISBN: 978-954-779-213-5. Retrieved from: http://www.matematikazv.weebly.com/uploads/1/2/3/ 8/12383653/matematika_8_klas_arhimed_2017_2018 .pdf [accessed: 17 October 2020].

[8]. Rivera, M. \& Maldonado, E. (2012). Elements of combinatorics in primary education. (in Spanish). En Sosa, Landy; Aparicio, Eddie, Rodríguez, Flor (Eds.), Memoria de la XV Escuela de Invierno en Matemática Educativa, 326-334. Ciudad de México: Red Cimates.

[9]. Rodriguez, R. (2015). The essentials in combinatorics with elements of statistics. Havana: Editorial University.

[10]. Todorov, V., \& Dimov, I. (2019, June). Efficient stochastic approaches for multidimensional integrals in Bayesian statistics. In International Conference on Large-Scale Scientific Computing (pp. 454-462). Springer, Cham.

[11]. Todorov, V., Dimov, I., \& Dimitrov, Y. (2018, October). Efficient quasi-Monte Carlo methods for multiple integrals in option pricing. In AIP Conference Proceedings (Vol. 2025, No. 1, p. 110007). AIP Publishing LLC.

[12]. Toncheva, N. \& Yordanova, I. (2007). A Historical Review on Teaching Probability and Statistics in the Bulgarian School from 1945 till 2005. Proceedings of the Thirty Sixth Spring Conference of the Union of Bulgarian Mathematicians St. Konstantin \& Elena resort, Varna, April 2-6, 2007.

[13]. Vasileva, M. (2008). Discrete structures - textbook. Shumen: Publishing house of the Ministry of Defense, National Military University "Vasil Levski", Faculty Artillery, ISBN 978-954-9681-32-1.

[14]. Veleva, E. (2014). The using of MS Excel in an introductory course of probability and statistics. Stochastics Education Problems, (1), 16-21.

[15]. Wilhelmi, R. (2004). Combinatorics and probability. (in Spanish). Grandas: GEEUG. Caps. 2 and 3. Retrieved from: https://www.ugr.es/ batanero/pages/ARTICULOS/lib rowhilhelmi.pdf [accessed: 13 November 2020].

[16]. Živanović, M. V., \& Pikula, M. T. (2013). Basic combinatorial configurations in preschool teacher education. Sinteze-časopis za pedagoške nauke, književnost i kulturu, 2(3), 23-31. 